\title{
G-ribo motif favors the formation of pseudoknots in ribosomal RNA
}

\author{
SERGEY V. STEINBERG and YURY I. BOUTORINE \\ Département de Biochimie, Université de Montréal, C.P. 6128, Succursale Centre-Ville, Montréal, QC H3C 3J7, Canada
}

\begin{abstract}
Analysis of the pseudoknots existing in the ribosomal RNA showed that four of them are formed with the help of G-ribo, a recently identified RNA recurrent motif. The analysis of these pseudoknots revealed two major aspects in the G-ribo motif structure, which together provide the structural context favoring the formation of two different types of pseudoknots. The first aspect pertains to a particular side-by-side juxtaposition of two double helices that facilitates switches of the polynucleotide chain between different strands. The second aspect deals with the presence of an adenosine at a specific place where it can stabilize a particular arrangement of two quasicoaxial helices required for the pseudoknot formation. Additional analysis shows that the latter aspect is also present in other pseudoknots not related to the G-ribo motif or the ribosome, and thus represents a general structural element favoring the formation of pseudoknots.
\end{abstract}

Keywords: G-ribo wrench; G-ribo ring; ribosome structure; RNA recurrent motif; RNA structure

\section{INTRODUCTION}

RNA pseudoknots can be defined as secondary structure arrangements in which a region within a hairpin loop forms a double helix with a region external to the hairpin proper (Gutell et al. 1994) (Supplemental Fig. 1). Pseudoknots are found in different RNA molecules and play a variety of different roles, which include the formation of the catalytic core of various ribozymes (Rastogi et al. 1996; Ke et al. 2004), self-splicing introns (Adams et al. 2004), and telomerase (Theimer et al. 2005). Pseudoknots also play critical roles in altering gene expression by inducing ribosomal frameshift and readthrough in different viruses (ten Dam et al. 1990; Shen and Tinoco 1995; Michiels et al. 2001; Egli et al. 2002; Nixon et al. 2002). Several pseudoknots are found in ribosomal RNA (Gutell et al. 1994), where they play potentially important structural and functional roles. The conditions for the formation of pseudoknots include the complementarity between the regions that are presumed to form the double helical stems as well as the proper lengths of the connector regions (Pleij et al. 1985). If the connectors are involved in specific interactions, their nucleotide sequences could also be

Reprint requests to: Sergey V. Steinberg, Département de Biochimie, Université de Montréal, C.P. 6128, Succursale Centre-Ville, Montréal, QC H3C 3J7, Canada; e-mail: serguei.chteinberg@umontreal.ca; fax: (514) 343-2210.

Article published online ahead of print. Article and publication date are at http://www.rnajournal.org/cgi/doi/10.1261/rna.495207. important. However, to which extent a broader structural context can affect the formation of a particular type of pseudoknot is mainly unknown.

In this paper, we show that G-ribo, a recently described RNA structural motif that pertains to a particular side-byside arrangement of two double helices (Steinberg and Boutorine 2007), mediates the formation of four different pseudoknots in the ribosomal RNA. Analysis of these cases reveals two aspects of the G-ribo motif that together determine the structural context favoring the pseudoknot formation. Both aspects facilitate the switches of the polynucleotide chain between different strands at the core of the pseudoknot structure. One aspect pertains to the specific juxtaposition of two double helices within the G-ribo motif, while the other one deals with the presence of an adenosine that can stabilize the particular arrangement of two quasicoaxial double helices. This adenosine stacks on top of one helix and forms an A-minor interaction with the last base pair of the other helix. Because almost identical arrangement is found in other pseudoknots not related to the G-ribo motif, its presence is thus considered an important factor promoting the formation of different pseudoknots.

\section{RESULTS}

\section{Background: the G-ribo motif}

The G-ribo motif represents a particular side-by-side arrangement of two double helices (Helices 1 and 2) 
connected by an unpaired region with at least three nucleotides (Steinberg and Boutorine 2007). The definitions of the helices, strands, layers, and particular nucleotides within the G-ribo motif are given in Figure 1A. At the zero layer, the top base pairs $[0 \mathrm{P} ; 0 \mathrm{Q}]$ and $[0 \mathrm{R} ; 0 \mathrm{~S}]$ of both helices are juxtaposed as shown in Figure 1B. At the center of this juxtaposition, guanosine $\mathrm{OP}$ forms two hydrogen bonds with the ribose of nucleotide $0 \mathrm{R}$. This interaction has given the name G-ribo to the whole arrangement. Other elements shared by all G-ribo motifs are the bulge between positions $0 \mathrm{R}$ and $+1 \mathrm{R}$ (T-bulge) and the nucleotide arrangements at layers +1 (not shown) and -1 (Fig. 1C). Together, these elements are able to fix the particular juxtaposition of Helices 1 and 2. Within the ribosome, the G-ribo motif has been found in eight different places: three in 16S rRNA and five in 23S rRNA.

One of the elements of the G-ribo motif that play an important role in the formation of the structures discussed in this paper is nucleotide $-1 \mathrm{~T}$, which is the last and, in most cases, the only nucleotide of the T-bulge (Fig. 1A).
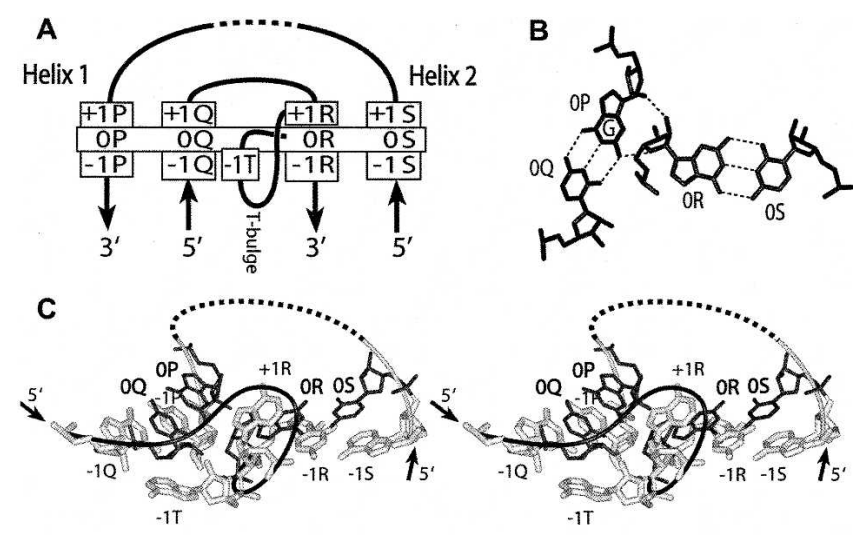

FIGURE 1. The general description of the G-ribo motif. (A) The secondary structure of the G-ribo motif. Helices 1 and 2 are, respectively, on the left and on the right. They are composed of, respectively, strands $\mathrm{P}$ and $\mathrm{Q}$ and strands $\mathrm{R}$ and $\mathrm{S}$. The dashed element indicates a possibility of a longer connector region between strands $\mathrm{S}$ and $\mathrm{P}$, which can include additional secondary structure elements. The name of each nucleotide consists of the number of the layer and of the letter that indicates the strand to which it belongs. The top base pairs of both helices $[0 \mathrm{P} ; 0 \mathrm{Q}]$ and $[0 \mathrm{R} ; 0 \mathrm{~S}]$ form the zero layer. For other layers within the helices, the numbering propagates in the negative direction. For the nucleotides of the connector regions that stack on top of the nucleotides of the zero layer, the numbers are positive. There is a bulge between nucleotides $0 \mathrm{R}$ and $+1 \mathrm{R}$ (the socalled T-bulge), which in most cases consists of only $1 \mathrm{nt}$ occupying position - 1T. $(B)$. The juxtaposition of base pairs $[0 \mathrm{P} ; 0 \mathrm{Q}]$ and [0R;0S]. Dashed lines stand for hydrogen bonds within and between the base pairs. In this juxtaposition, the ribose of $0 \mathrm{R}$ interacts with the ribose and the base of $0 \mathrm{P}$. To make this interaction possible, $0 \mathrm{P}$ should be guanosine. $(C)$. The tertiary structure of the G-ribo motif $S 861$ from the E. coli ribosome (Schuwirth et al. 2005). The nucleotides forming the zero layer are black. Other nucleotides are white. At layer +1 , only nucleotide $+1 \mathrm{R}$ is shown. Nucleotide $-1 \mathrm{~T}$, which is predominantly adenosine, forms the A-minor interaction (Doherty et al. 2001; Nissen et al. 2001) with base pair [-1P;- 1Q].
Nucleotide $-1 \mathrm{~T}$ is predominantly adenosine, and it forms an A-minor interaction (Doherty et al. 2001; Nissen et al. 2001) with base pair [-1P;-1Q] (Fig. 1A). One side of the $-1 \mathrm{~T}$ base stacks to the ribose of $0 \mathrm{Q}$, while the other side is open for interaction with a nucleotide from layer -2 . As we argue here, such a special position of nucleotide $-1 \mathrm{~T}$ is responsible for the ability of the G-ribo motif to promote the formation of pseudoknots.

\section{Chain break in strand $\mathbf{P}$}

As mentioned above, the interactions that keep the particular juxtaposition of Helices 1 and 2 within the G-ribo motif are spread over three layers between +1 and -1 . Below layer -1 , the helices become completely separated, which presumes that no elements specific to the G-ribo motif exist in this zone. However, at layer - 2, most G-ribo motifs share a new unusual element. In particular, we noticed that in five motifs there is a break in strand $\mathrm{P}$ between positions $-1 \mathrm{P}$ and $-2 \mathrm{P}$, so that the two positions become separated in the polynucleotide chain by at least a dozen nucleotides, and in some cases, by hundreds of nucleotides (Fig. 2; Supplemental Fig. 2). Unlike strand P, strand Q in all G-ribo motifs continues between layers - 1 and -2 without interruption. The presence of a noninterrupted Q strand allows Helix 1 to continue below layer -1 , while the break of the chain in strand $\mathrm{P}$ provides new opportunities for connections between different strands of the G-ribo motif. Further analysis showed that in all five cases of the G-ribo motif where positions $-1 \mathrm{P}$ and $-2 \mathrm{P}$ are distant from each other in the polynucleotide chain, $-1 \mathrm{P}$ is connected to a nucleotide of a lower layer of strand $\mathrm{Q}$, while $-2 \mathrm{P}$ is connected to a nucleotide of either strand $\mathrm{Q}$ or $\mathrm{S}$. Each of the two types of the $-2 \mathrm{P}$ connection corresponds to a particular type of pseudoknot.

\section{Wrench pseudoknot in L1024}

We will first consider motif L1024, whose secondary structure is shown in Figure 2B. Also, for convenience, the representation of the same motif as it is presented in the standard secondary structure of $23 \mathrm{~S}$ rRNA can be seen in Supplemental Figure 2A. In L1024, nucleotide - 1P (G1025) is connected to - 4Q (G1136) with the help of a long connector region. Most of this region has no contacts with other parts of the L1024 G-ribo motif and is irrelevant for its structure, while for a proper connection between $-1 \mathrm{P}$ and $-4 \mathrm{Q}$, one nucleotide is sufficient. Also, in L1024, $-2 \mathrm{P}(\mathrm{C} 1005)$ is directly connected to $-7 \mathrm{~S}$ (U1004), while $-4 \mathrm{P}$ (C1007) is connected to - 6S (G1011) with the help of a 3-nucleotide (nt) connector. In the secondary structure of this arrangement, region 1005-1010, encompassing $3 \mathrm{nt}$ of strand $\mathrm{P}$ followed by three unpaired adenosines, is a bulge of strand $\mathrm{S}$ between positions $-6 \mathrm{~S}$ and $-7 \mathrm{~S}$. The formation of the three base pairs between 
nucleotides $1005-1007$ and 1136-1138 creates a pseudoknot. For this reason, region 1005-1010 bulging of strand S is henceforth called the pseudoknot bulge. Due to the visible similarity to a wrench, the whole arrangement encompassing Helices 1 and 2 and the connector regions between them excluding the long connector between $-1 \mathrm{P}$ and $-4 \mathrm{Q}$ will be called the G-ribo wrench.

Analysis of the L1024 G-ribo wrench revealed additional details of its tertiary structure relevant for the pseudoknot formation. Thus, compared to the standard A-RNA conformation, base pair $[-2 \mathrm{P} ;-2 \mathrm{Q}]$ is substantially displaced with respect to base pair $[-1 \mathrm{P} ;-1 \mathrm{Q}]$ (Fig. 3A,B). The displacement can be represented as a combination of a rotation around atom $\mathrm{O}^{\prime}$ ' of nucleotide $-2 \mathrm{Q}$ for about $50^{\circ}$ and of an additional translation for about $4 \AA$. For $-2 \mathrm{Q}$, the total displacement is about $5 \AA$, which allows this nucleotide to maintain its stacking to $-1 \mathrm{Q}$. Nucleotide $2 \mathrm{P}$, however, has moved for $>10 \AA$ and has lost all its contacts with $-1 \mathrm{P}$. Such a movement of base pair $[-2 \mathrm{P} ;-2 \mathrm{Q}]$ brings it closer to Helix 2, thus allowing the two consecutive nucleotides - 7S (U1004) and - 2P (C1005) to be simultaneously involved in base pairing within the two different Helices 1 and 2. Needless to say, this movement has become possible due to the absence of the covalent link between $-1 \mathrm{P}$ and $-2 \mathrm{P}$.

Additional interactions within the G-ribo wrench can stabilize this arrangement. First, an unpaired uridine $-5 \mathrm{Sa}$ (U1012), which is bulged of strand S between nucleotides $-6 \mathrm{~S}$ and $-5 \mathrm{~S}$ (Fig. 2A,B), forms a Hoogsteen base pair with adenosine $-1 \mathrm{~T}$. Within this base pair, $-1 \mathrm{~T}$ and $-5 \mathrm{Sa}$ tightly bind nucleotide $-2 \mathrm{P}$ : while $-1 \mathrm{~T}$ stacks to the base of $-2 \mathrm{P},-5 \mathrm{Sa}$ interacts with its ribose (Fig. 3B). This interaction effectively stabilizes the displaced position of nucleotide $-2 \mathrm{P}$. Also, the last two adenosines A1009-A1010 of the pseudoknot bulge are involved in the A-minor interactions (Doherty et al. 2001; Nissen et al. 2001) with base pairs C1153-G1002 and C1152-G1003 occupying, respectively, layers -9 and -8 of Helix 2 (Fig. 2B).

On the level of the tertiary structure, the L1024 G-ribo wrench represents a compact globular domain with dimensions $26 \AA \times 34 \AA \times 38 \AA$ attached to the rest of Helix 2 (Supplemental Fig. 3A). The compactness of the G-ribo wrench and its saturation with secondary and tertiary interactions suggests that it is stable on its own and can form independently of the rest of the ribosome structure.

\section{Wrench pseudoknot in $\mathbf{S 8 6 1}$}

Another wrench pseudoknot with a very similar structure is associated with motif S861 (Fig. 2C; Supplemental Fig. 2B). The most essential difference of S861 from L1024 pertains to the length of the pseudoknot bulge, which contains $5 \mathrm{nt}$ instead of six. The superposition of the S861 structure with that of L1024 identifies the missing nucleotide as $-4 \mathrm{P}$, which leaves the duplex between strand $\mathrm{Q}$ and the pseudoknot bulge with only two base pairs. Other differences of S861 from L1024 deal with the absence of the long insertion between positions $-1 \mathrm{P}$ and $-3 \mathrm{Q}$ and with the presence of long insertions between $+1 \mathrm{~S}$ and $+1 \mathrm{P}$ and between $-6 \mathrm{~S}$ and $-5 \mathrm{~S}$. None of these insertions is relevant to the structure of the S861 pseudoknot. Nucleotide U820 in S861, the last one in the insertion between $-6 \mathrm{~S}$ and $-5 \mathrm{~S}$, is equivalent to $-5 \mathrm{Sa}$ in L1024: it occupies the same position just before $-5 \mathrm{~S}$ and has the same uridine identity. It also forms a Hoogsteen base pair with adenosine $-1 \mathrm{~T}$ and stacks to the ribose of $-2 \mathrm{P}$ (Fig. 3C).

The superposition of the structures of the L1024 and S861 pseudoknots demonstrates a very strong similarity over 11 layers from +1 to -9 , i.e., until the end of Helix 2 in both motifs (Supplemental Fig. 3B).

\section{Wrench pseudoknot in S521}

Within the ribosome structure, there is one more wrench pseudoknot that associates with motif S521 (Fig. 2D; Supplemental Fig. 2C). In this pseudoknot, like in L1024,

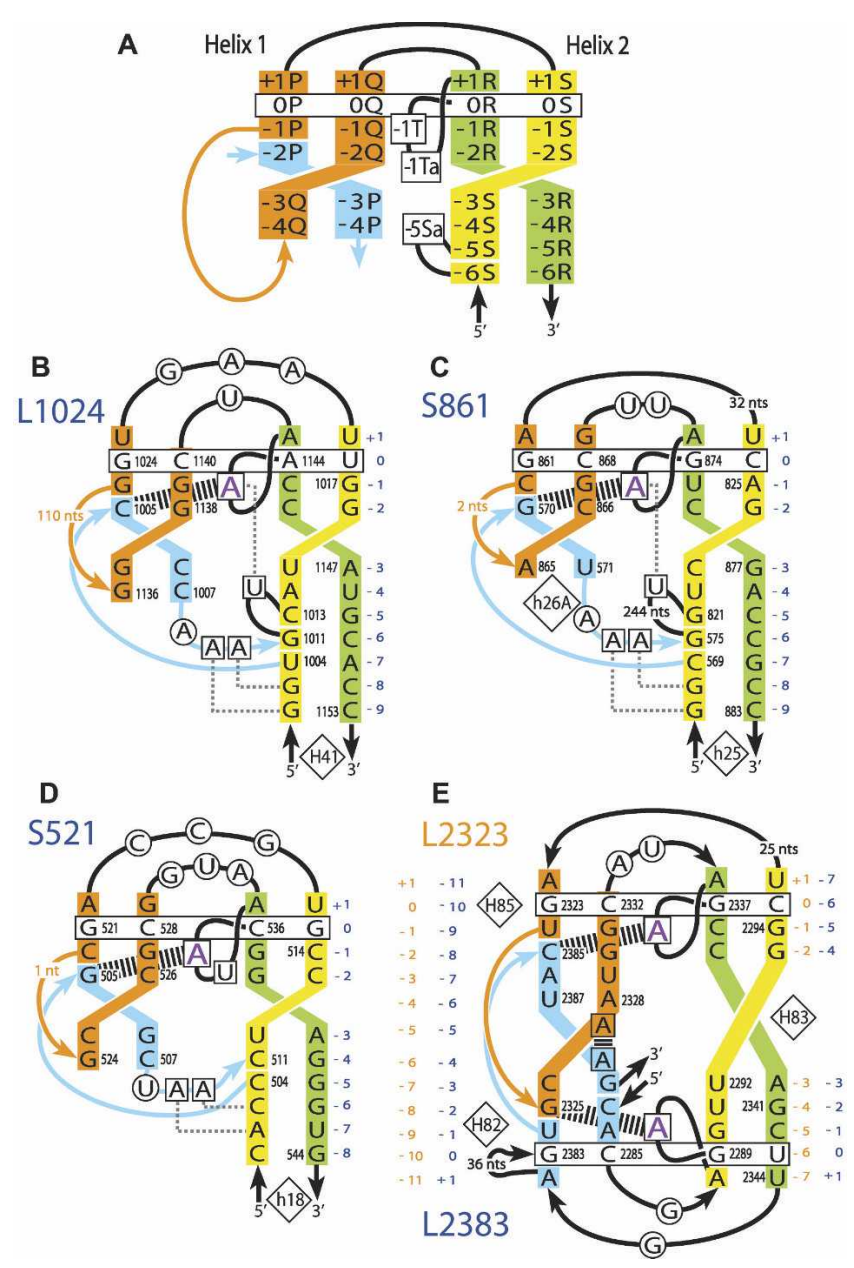

FIGURE 2. (Legend on next page) 
the pseudoknot bulge contains $6 \mathrm{nt}$ and forms three base pairs with strand Q. Unlike L1024 and S861, S521 does not contain any long insertion and is thus built of only one piece of 16S rRNA between nucleotides 502 and 543 .

Some aspects of the structure of the S521 pseudoknot make it different from those of L1024 and S861. In particular, the pseudoknot bulge in S521 is inserted between positions $-4 S$ and $-5 S$ of the $S$ strand, rather than between $-6 \mathrm{~S}$ and $-7 \mathrm{~S}$. Also, the last two adenosines of the pseudoknot bulge form the A-minor interactions with base pairs U543-A502 and G542-C503, which occupy, respectively, layers -7 and -6 of Helix 2, and not -9 and -8 . In other words, in S521, compared to L1024 and S861, the position of the pseudoknot bulge in strand $\mathrm{S}$ as well as of the A-minor interactions between the pseudoknot bulge and Helix 2 is shifted for two layers up. Analysis shows that due to this shift, Helices 1 and 2 in S521 become displaced with

FIGURE 2. The secondary structures of the four G-ribo-based pseudoknots in the E. coli rRNA. (A) The template used for depicting the secondary structures. The base pairs at the zero layer are enclosed in the horizontally oriented rectangle. In all pseudoknots, there is a break of the polynucleotide chain in strand $\mathrm{P}$ between positions - $1 \mathrm{P}$ and $-2 \mathrm{P}$. Nucleotide $-1 \mathrm{P}$ is connected to a lower layer of strand $\mathrm{Q}$, thus forming a stem-loop structure (orange) in Helix 1. Depending on the type of pseudoknot, region $[-2 \mathrm{P} ;-4 \mathrm{P}]$ (cyan) is connected to different parts of the structure. Strands R and S of Helix 2 are, respectively, green and yellow. Nucleotide $-1 \mathrm{Ta}$ exists only in S521, while nucleotide - 5Sa exists only in L1024 and S861. (B-E) In all structures, the first letter in the name of a motif ( $\mathrm{S}$ or L) stands for the ribosomal subunit, small or large, in which the motif has been found. The number in the name corresponds to that of nucleotide $0 \mathrm{P}$ in the standard E. coli numeration of rRNA. The numbers of the helices in the standard $16 \mathrm{~S}$ and $23 \mathrm{~S}$ rRNA secondary structures are shown in diamonds. The unpaired nucleotides that are important for the integrity of the pseudoknots are squared. Adenosine - 1T is shown in magenta. All other nucleotides outside the double helical regions are circled. The zebra bands connecting positions $-2 \mathrm{P}$ and $-1 \mathrm{~T}$ as well as adenosines A2327 and A2386 (in E) indicate the stacking interactions essential for the integrity of the arrangement. $(B-D)$ Different G-ribo wrenches. In these structures, nucleotides $-2 \mathrm{P},-$ $3 \mathrm{P}$, and $-4 \mathrm{P}(-4 \mathrm{P}$ exists only in $B$ and $D)$ belong to the pseudoknot bulge (cyan). The pseudoknot bulge is inserted into strand $\mathrm{S}$ either between positions - 7S and - 6S (L1024 and S861) or between positions - 5S and - 4S (S521). The numbers of layers in both Helices 1 and 2 are shown in blue. The dashed lines connecting the two last adenosines of the pseudoknot bulge with the base pairs in Helix 2 indicate the A-minor interactions (Doherty et al. 2001; Nissen et al. 2001). The dashed line connecting adenosine $-1 \mathrm{~T}$ and uridine - 5Sa (only in L1024 and S861) stands for the Hoogsteen base pair. (E) The G-ribo ring. The structure is formed by two symmetrically positioned G-ribo motifs L2323 and L2383. The numbers of layers are provided individually for each helix and for each motif. With respect to motifs L2323 and L2383, the numbers are, respectively, blue and orange. Strand P in L2323 (orange in the upper part, cyan in the lower part) becomes strand Q in L2383. Strand Q in L2323 (cyan in the upper part, orange in the lower part) becomes strand P in L2383. In L2383, the cyan stem-loop plays the same role as the orange stemloop in L2323. The two loops, orange and cyan, form the kissing interactions within Helix 1. Within the kissing helix, adenosines A2327 and A2386 are not involved in base pairing and stack to each other. The green and yellow strands are, respectively, strands R and S in L2323 and strands S and R in L2383.
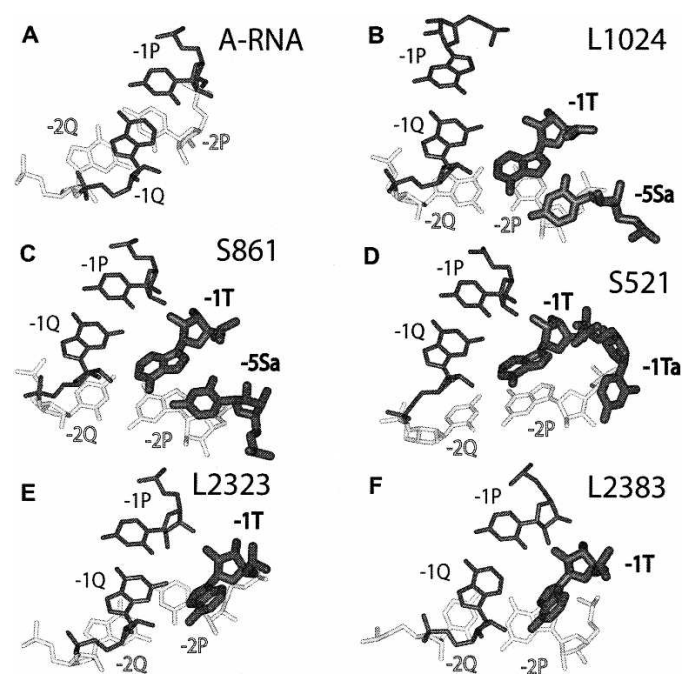

FIGURE 3. The displacement of base pair [-2P;-2Q] with respect to $[-1 \mathrm{P} ;-1 \mathrm{Q}]$ in the G-ribo-based pseudoknots. All structures are aligned by the position of nucleotide $-1 \mathrm{Q}$. Nucleotides $-2 \mathrm{P}$ and $2 \mathrm{Q}$ are white, while all other nucleotides are black. Nucleotides - 1T, - 5Sa (only in L1023 and S861), and - 1Ta (only in S521), which are involved in the stabilization of the position of nucleotide $-2 \mathrm{P}$, are shown thick. Compared to the juxtaposition of two consecutive base pairs in a regular RNA double helix $(A)$, base pair $[-2 \mathrm{P} ;-2 \mathrm{Q}]$ in the G-ribo wrenches $(B-D)$ is rotated for about $50^{\circ}$ around atom $\mathrm{O}^{\prime}$ of $-2 \mathrm{Q}$ in the direction of the minor groove and is additionally shifted for about $4 \AA$. In motifs L2323 and L2383 forming the G-ribo ring $(E, F)$, the displacement of base pair $[-2 \mathrm{P} ;-2 \mathrm{Q}]$ is smaller than in the G-ribo wrenches, and in L2323 (E), it is smaller than in L2383 (F). Due to this displacement, $-2 \mathrm{P}$ in all pseudoknots has lost its interaction with $-1 \mathrm{P}$ and becomes stacked to $-1 \mathrm{~T}$. Additional stabilization of the $-2 \mathrm{P}$ position in the G-ribo wrenches is provided through the interaction of its ribose with either $-5 \mathrm{Sa}$ or $-1 \mathrm{Ta}$. Uridine - 5Sa, when it exists, forms a Hoogsteen base pair with adenosine $-1 \mathrm{~T}$.

respect to each other for a few angstroms compared to their juxtaposition in L1024 and S861 (not shown). As a result, pseudoknot S521 is superposable with neither L1024 nor S861, although it is still very close to both these structures.

Another difference between the S521 pseudoknot and those of L1024 and S861, which seems to be linked to the displacement of the pseudoknot bulge, consists in the absence of the bulged nucleotide - 5Sa. Indeed, due to the new position of the pseudoknot bulge, its interaction with strand Q would have interfered with the formation of base pair [-5Sa; $-1 \mathrm{~T}]$. Interestingly, the S521 pseudoknot contains another unusual element, a second nucleotide in the T-bulge immediately before - 1T (nucleotide - 1Ta [U534]; Fig. 2D), the presence of which could compensate for the absence of uridine $-5 \mathrm{Sa}$. Like $-5 \mathrm{Sa}$, nucleotide $-1 \mathrm{Ta}$ stacks to the ribose of $-2 \mathrm{P}$, and thus would stabilize the position of the latter in the situation when -5 Sa does not exist due to the displacement of the pseudoknot bulge (Fig. 3D).

Analysis of the available nucleotide sequences of $16 \mathrm{~S}$ rRNA (Wuyts et al. 2004) showed that in archaea, unlike in bacteria, the pseudoknot bulge of S521 is integrated into 
the S-stem between positions - 3S and $-4 \mathrm{~S}$ and contains either 7 or 8 nt (Supplemental Fig. 4). However, our preliminary data show that such modifications are local and do not affect the global pseudoknot conformation.

\section{Ring pseudoknot}

In the ribosomal RNA, there is another G-ribo-based pseudoknot whose structure, however, is essentially different from that of the wrench pseudoknots. This new pseudoknot is built of fragment 2283-2389 of 23S rRNA and is based on a specific arrangement of three consecutive double helices 82,83 , and 85 of the $23 \mathrm{~S}$ rRNA secondary structure. The juxtaposition of helices 82 and 83 and of helices 83 and 85 is mediated by G-ribo motifs L2383 and L2323, respectively (Fig. 2E; Supplemental Fig. 2D). The whole arrangement is stabilized by the kissing interaction between the unpaired region adjacent to Helix 82 and the loop-closing Helix 85 . This kissing interaction constitutes a pseudoknot. The four double helical regions, i.e., Helices 82, 83, 85, and the kissing helix, together form a circular almost ideally symmetric structure, which we call the G-ribo ring. In three dimensions, this structure looks like a compact disk of $\sim 40 \AA$ in diameter and $20 \AA$ in width (Supplemental Fig. 3C).

In the secondary structure of the G-ribo ring, the $\mathrm{P}$ and R stems of L2323 become, respectively, the Q and S stems of L2383 and vice versa. In both motifs, as in those forming the wrench pseudoknots, the $\mathrm{P}$ strand is interrupted between positions - $1 \mathrm{P}$ and $-2 \mathrm{P}$. However, unlike in G-ribo wrenches, in both motifs L2323 and L2383, nucleotides $-1 \mathrm{P}$ and $-2 \mathrm{P}$ are directly connected to nucleotides $-8 \mathrm{Q}$ and $-9 \mathrm{Q}$, respectively. Due to the symmetry of the arrangement, positions $-1 \mathrm{P}$ and $-2 \mathrm{P}$ in one motif become, respectively, positions $-9 \mathrm{Q}$ and $-8 \mathrm{Q}$ in the other motif. Correspondingly, the connection between $-1 \mathrm{P}$ and $-8 \mathrm{Q}$ in L2323 becomes the connection between $-2 \mathrm{P}$ and - 9Q in L2383 and vice versa. For the kissing double helix, each G-ribo motif donates its Q strand, which would form a double helix with the $\mathrm{P}$ strand provided by the other G-ribo motif. If one determines the length of each helix as the number of layers from the zero layer in one G-ribo motif to the zero layer in the other motif, Helices 1 and 2 contain 11 and 7 layers, respectively.

The ability of the G-ribo ring to form the kissing helix depends on the complementarity of the kissing regions and on their proper juxtaposition. The latter, in turn, depends on the structure of all elements between the kissing regions all along the G-ribo ring, which would together guarantee that these regions become proximal to each other in the orientation prone for the formation of a double helix. Analysis shows that within the G-ribo ring, there are four elements whose structure has been tuned in order to reach the proper juxtaposition of the kissing regions. Because both motifs L2323 and L2383 are rigidly attached to Helix 2, their juxtaposition strongly depends on its length. A deletion or an insertion of a base pair in Helix 2 would displace one kissing region with respect to the other for $\sim 15 \AA$, which makes manipulations with the length of Helix 2 an effective but rather rough exercise, and may require an additional smoother tuning in other parts of the G-ribo ring. Such a tuning is provided through the displacement between base pairs [-2P; - 2Q] and [-1P; - 1Q] in both motifs L2323 and L2383 (Fig. 3E,F). In these motifs, like in those forming the wrench arrangements, the chain break between positions $-1 \mathrm{P}$ and $-2 \mathrm{P}$ allows a displacement of base pair $[-2 \mathrm{P}$; $-2 \mathrm{Q}]$ with respect to $[-1 \mathrm{P} ;-1 \mathrm{Q}]$ to the extent that $-2 \mathrm{P}$ becomes stacked to $-1 \mathrm{~T}$. This displacement facilitates the formation of the kissing helix between the -1 layers of both G-ribo motifs. Compared to the wrench arrangements, the displacement of base pair [ $-2 \mathrm{P} ;-2 \mathrm{Q}]$ in motifs L2323 and L2383 is smaller, which indicates the existence of some flexibility in this region. This flexibility allows the juxtaposition of base pairs $[-1 \mathrm{P} ;-1 \mathrm{Q}]$ and $[-2 \mathrm{P} ;-2 \mathrm{Q}]$ to be tuned to the requirements imposed by the particular type of pseudoknot.

The structure of the G-ribo ring demonstrates that with a proper choice of the length of Helix 2 and of the juxtapositions of base pairs $[-2 \mathrm{P} ;-2 \mathrm{Q}]$ and $[-1 \mathrm{P}$; - 1Q] in both motifs L2323 and L2383 the two kissing regions can be brought close to each other. It may, however, be more difficult to achieve an arrangement in which Helix 85, the kissing helix, and Helix 82 form together a coaxially stacked domain. As one can see in Figure 2E, there is mismatch A2327-A2388 in the middle of the kissing double helix. In the tertiary structure, the two adenosines, instead of forming a base pair, occupy neighboring layers and stack to each other. The presence of an unpaired region in the middle of the kissing helix provides an additional flexibility to the latter, which allows the formation of a bend for about $65^{\circ}$ between A2388 and base pair A2326-U2389 (Supplemental Fig. 3C). Without such a bend, the closure of the G-ribo ring seems to be essentially more difficult if possible at all, even though the kissing loops remain proximal to each other due to the proper choice of the other aspects discussed above. The bend in the kissing helix is stabilized by the A-minor interactions (Doherty et al. 2001; Nissen et al. 2001) of the two adenosines A2327-A2388 with Helix 81 of 23S rRNA (not shown). This interaction constitutes the only contact made by an element of the G-ribo ring with the rest of $23 \mathrm{~S}$ rRNA. As in the case of the G-ribo wrenches, the compactness of the G-ribo ring and its saturation with secondary and tertiary interactions suggest that it can be stable independently of other parts of the ribosome.

\section{Evolutionary conservation of the G-ribo-based pseudoknots}

All identified G-ribo-based pseudoknots are present in the ribosomes of all prokaryotic organisms. This conclusion is 
based on the following observations. First, all four pseudoknots discussed here had been predicted as universal secondary structure elements based on comparative analysis of the nucleotide sequences of bacterial and archaeal ribosomal RNA long before the elucidation of the ribosome tertiary structure (Gutell et al. 1994). We now know that these pseudoknots are formed with the help of the G-ribo motif, all examples of which within the ribosome were recently shown to be highly conserved in all prokaryotes (Steinberg and Boutorine 2007).

The structures of the pseudoknots are also highly conserved. Analysis of the available nucleotide sequences of ribosomal RNA (Wuyts et al. 2004) shows that except for the above-mentioned variation in the S521 pseudoknot between bacteria (Fig. 2D; Supplemental Fig. 2C) and archaea (Supplemental Fig. 4) the secondary structures of all other pseudoknots are the same in all prokaryotic organisms. Also, all G-ribo-based pseudoknots have virtually identical conformations in all available high-resolution structures of the ribosome and of its subunits (Schluenzen et al. 2000; Wimberly et al. 2000; Harms et al. 2001; Nissen et al. 2001; Schuwirth et al. 2005; Korostelev et al. 2006; Selmer et al. 2006), including that of the archaeal 50S subunit (Nissen et al. 2001).

The level of conservation of some tertiary elements of the pseudoknot structure also deserves mentioning (Supplemental Table 1). Thus, in all G-ribo motifs, the predominant identity of nucleotide $-1 \mathrm{~T}$ is adenosine (Wuyts et al. 2004). However, for different G-ribo motifs, the level of conservation varies between $54 \%$ and $100 \%$. Only in the three motifs corresponding to the G-ribo wrenches this level is approaching or equal to $100 \%$. So a high level of conservation is understandable in view of the special role played by $-1 \mathrm{~T}$ in providing a strong displacement of base pair $[-2 \mathrm{P} ;-2 \mathrm{Q}]$ with respect to $[-1 \mathrm{P} ;-1 \mathrm{Q}]$ observed in the wrench pseudoknots.

Uridine in position - 5Sa exists in almost $100 \%$ of all prokaryotic sequences of S861, while in L1024, it is conserved only in archaea. In bacteria, on the contrary, position - 5Sa in L1024 is occupied by uridine only in $87.5 \%$ of the cases, while in $9 \%$ of the sequences nucleotide $-5 \mathrm{Sa}$ is cytidine. The cytidine identity of $-5 \mathrm{Sa}$ allows this nucleotide to form the Hoogsteen base pair with adenosine - 1T, which would be rather similar to the Hoogsteen UA base pair formed if $-5 \mathrm{Sa}$ is uridine. Compared to the uridine - 5Sa in L1024 and S861, the uridine - 1Ta in S521 is less conserved, which reflects the fact that this nucleotide, unlike $-5 \mathrm{Sa}$, is not involved in formation of specific hydrogen bonds with $-1 \mathrm{~T}$, so that the constraints imposed on its identity can be less restrictive.

In the G-ribo motifs composing the G-ribo ring, the identity of $-1 \mathrm{~T}$ is less restricted to adenosine than in the G-ribo wrenches, seemingly, because of a smaller displacement of base pair $[-2 \mathrm{P} ;-2 \mathrm{Q}]$, which does not require that the interaction between $-2 \mathrm{P}$ and $-1 \mathrm{~T}$ be very stable, and also because of the absence of specific interactions equivalent to that between $-1 \mathrm{~T}$ and $-5 \mathrm{Sa}$ in L1024 and S861. Another element of the structure of the G-ribo ring built of adenosines A2327-A2388 is conserved at the level of $100 \%$ both in bacteria and archaea, reflecting its pivotal role in the whole arrangement.

To conclude, not only all G-ribo-based pseudoknots are highly conserved, but also conserved are the structural elements important for their formation. Deviations from the predominant identities are allowed only for those elements whose variation does not compromise the integrity of the arrangements.

\section{DISCUSSION}

The results presented here clearly position the G-ribo motif as pseudoknot prone. Indeed, out of eight G-ribo motifs identified in the ribosome, five are involved in the pseudoknot formation. Also, within the ribosomal RNA, there are 10 pseudoknots in which the characteristic secondary structure elements contain at least two base pairs (Gutell et al. 1994), and the G-ribo-based pseudoknots account for almost a half of them. An important feature of the G-ribo motif related to its proneness toward pseudoknots is the side-by-side juxtaposition of Helices 1 and 2. In the G-ribo wrenches, this juxtaposition facilitates the covalent reconnections between strands $\mathrm{P}$ and $\mathrm{S}$. In the G-ribo ring, the side-by-side arrangement of Helices 82 and 83 as well as of Helices 83 and 85 mediated by two G-ribo motifs L2383 and L2323 brings the two kissing regions close to each other.

The particular juxtaposition of Helices 1 and 2 within the G-ribo motif is not, however, the only feature of its structure that may be relevant for the pseudoknot formation: all G-ribo-based pseudoknots contain a chain break between positions $-1 \mathrm{P}$ and $-2 \mathrm{P}$. In the G-ribo ring, even the second chain break between positions $-8 \mathrm{Q}$ and $-9 \mathrm{Q}$ is, in fact, one between $-1 \mathrm{P}$ and $-2 \mathrm{P}$, if the second G-ribo motif is taken as a reference point. Such a universal position of this chain break is rather surprising, given that the wrench and ring pseudoknots in many aspects are very different.

An important aspect of the chain break between - 1P and $-2 \mathrm{P}$ is that it allows an additional tuning in the position of the lower part of Helix 1, thus facilitating the other reconnections of the polynucleotide chain between the strands. Although this tuning is important for pseudoknot formation, it cannot explain the universal position of the break between $-1 \mathrm{P}$ and $-2 \mathrm{P}$, because a break between $0 \mathrm{P}$ and $-1 \mathrm{P}$ or between $-2 \mathrm{P}$ and $-3 \mathrm{P}$ would allow similar movements of Helix 1 toward Helix 2. The uniqueness of the break between $-1 \mathrm{P}$ and $-2 \mathrm{P}$ seems to be linked to the presence of adenosine - $1 \mathrm{~T}$, the interaction with which would favor the displacement of nucleotide $-2 \mathrm{P}$. Thus, the particular location of nucleotide $-1 \mathrm{~T}$ and its openness for interaction with a nucleotide of 
layer -2 would favor the formation of arrangements with a chain break between $-1 \mathrm{P}$ and $-2 \mathrm{P}$ and with a displacement of nucleotide $-2 \mathrm{P}$ toward Helix 2 .

While nucleotide $-2 \mathrm{P}$ stacks to $-1 \mathrm{~T}$, its position still remains flexible and can adapt to the requirements of the particular type of pseudoknot. However, the stacking with $-1 \mathrm{~T}$ per se suffices the promotion of only a relatively mild displacement of $-2 \mathrm{P}$ like that present in the G-ribo ring. For a stronger displacement observed in the G-ribo wrenches, an additional stabilization of the $-2 \mathrm{P}$ position through the contact of its ribose with either $-5 \mathrm{Sa}$ or $-1 \mathrm{Ta}$ is required.

Finally, we would like to compare the G-ribo-based pseudoknots with other known pseudoknots. Our inspection of different RNA structures showed that the particular arrangement of base pairs $[-1 \mathrm{P} ;-1 \mathrm{Q}]$ and $[-2 \mathrm{P} ;-2 \mathrm{Q}]$ stabilized by adenosine $-1 \mathrm{~T}$, which is at the core of all four pseudoknots discussed in this paper, exists also in other pseudoknots, unrelated to the G-ribo motif, both in and outside the ribosome (Supplemental Fig. 5). We can thus conclude that this arrangement, which facilitates the particular type of chain switch between two quasicoaxial double helices, is an essential part of the structural contexts favoring the formation of different kinds of pseudoknots. From this point of view, the G-ribo motif represents a particular way of the polynucleotide chain arrangement around the central part that would provide for a compact and stable structure suited to a particular function.

\section{SUPPLEMENTAL DATA}

All Supplemental material and figures are available at http:// www.esi.umontreal.ca/chteinbe/publications/supplement.html.

\section{ACKNOWLEDGMENTS}

The authors are grateful to Dr. Lea Brakier-Gingras for important discussions. S.V.S. acknowledges a grant from CIHR and fellowships from CIHR and FRSQ.

Received February 6, 2007; accepted April 17, 2007.

\section{REFERENCES}

Adams, P.L., Stahley, M.R., Kosek, A.B., Wang, J., and Strobel, S.A. 2004. Crystal structure of a self-splicing group I intron with both exons. Nature 430: 45-50.

Doherty, E.A., Batey, R.T., Masquida, B., and Doudna, J.A. 2001. A universal mode of helix packing in RNA. Nat. Struct. Biol. 8: 339343.

Egli, M., Minasov, G., Su, L., and Rich, A. 2002. Metal ions and flexibility in a viral RNA pseudoknot at atomic resolution. Proc. Natl. Acad. Sci. 99: 4302-4307.
Gutell, R.R., Larsen, N., and Woese, C.R. 1994. Lessons from an evolving rRNA: $16 \mathrm{~S}$ and $23 \mathrm{~S}$ rRNA structures from a comparative perspective. Microbiol. Rev. 58: 10-26.

Harms, J., Schluenzen, F., Zarivach, R., Bashan, A., Gat, S., Agmon, I., Bartels, H., Franceschi, F., and Yonath, A. 2001. High-resolution structure of the large ribosomal subunit from a mesophilic eubacterium. Cell 107: 679-688.

Ke, A., Zhou, K., Ding, F., Cate, J.H., and Doudna, J.A. 2004. A conformational switch controls hepatitis delta virus ribozyme catalysis. Nature 429: 201-205.

Korostelev, A., Trakhanov, S., Laurberg, M., and Noller, H.F. 2006. Crystal structure of a $70 \mathrm{~S}$ ribosome-tRNA complex reveals functional interactions and rearrangements. Cell 126: 1065-1077.

Michiels, P.J., Versleijen, A.A., Verlaan, P.W., Pleij, C.W., Hilbers, C.W., and Heus, H.A. 2001. Solution structure of the pseudoknot of SRV-1 RNA, involved in ribosomal frameshifting. J. Mol. Biol. 310: 1109-1123.

Nissen, P., Ippolito, J.A., Ban, N., Moore, P.B., and Steitz, T.A. 2001. RNA tertiary interactions in the large ribosomal subunit: The A-minor motif. Proc. Natl. Acad. Sci. 98: 4899-4903.

Nixon, P.L., Rangan, A., Kim, Y.G., Rich, A., Hoffman, D.W., Hennig, M., and Giedroc, D.P. 2002. Solution structure of a luteoviral P1-P2 frameshifting mRNA pseudoknot. J. Mol. Biol. 322: 621-633.

Pleij, C.W., Rietveld, K., and Bosch, L. 1985. A new principle of RNA folding based on pseudoknotting. Nucleic Acids Res. 13: 17171731.

Rastogi, T., Beattie, T.L., Olive, J.E., and Collins, R.A. 1996. A longrange pseudoknot is required for activity of the Neurospora VS ribozyme. EMBO J. 15: 2820-2825.

Schluenzen, F., Tocilj, A., Zarivach, R., Harms, J., Gluehmann, M., Janell, D., Bashan, A., Bartels, H., Agmon, I., Franceschi, F., et al. 2000. Structure of functionally activated small ribosomal subunit at $3.3 \AA$ resolution. Cell 102: 615-623.

Schuwirth, B.S., Borovinskaya, M.A., Hau, C.W., Zhang, W., Vila-Sanjurjo, A., Holton, J.M., and Cate, J.H. 2005. Structures of the bacterial ribosome at $3.5 \AA$ resolution. Science 310: $827-$ 834.

Selmer, M., Dunham, C.M., Murphy, F.V.T., Weixlbaumer, A., Petry, S., Kelley, A.C., Weir, J.R., and Ramakrishnan, V. 2006. Structure of the 70S ribosome complexed with mRNA and tRNA. Science 313: 1935-1942.

Shen, L.X. and Tinoco Jr., I. 1995. The structure of an RNA pseudoknot that causes efficient frameshifting in mouse mammary tumor virus. J. Mol. Biol. 247: 963-978.

Steinberg, S.V. and Boutorine, Y.I. 2007. G-ribo: A new structural motif in ribosomal RNA. RNA 13: 549-554.

ten Dam, E.B., Pleij, C.W., and Bosch, L. 1990. RNA pseudoknots: Translational frameshifting and readthrough on viral RNAs. Virus Genes 4: 121-136.

Theimer, C.A., Blois, C.A., and Feigon, J. 2005. Structure of the human telomerase RNA pseudoknot reveals conserved tertiary interactions essential for function. Mol. Cell 17: 671682.

Wimberly, B.T., Brodersen, D.E., Clemons Jr., W.M., MorganWarren, R.J., Carter, A.P., Vonrhein, C., Hartsch, T., and Ramakrishnan, V. 2000. Structure of the 30S ribosomal subunit. Nature 407: 327-339.

Wuyts, J., Perriere, G., and Van De Peer, Y. 2004. The European ribosomal RNA database. Nucleic Acids Res. 32: D101-D103. 

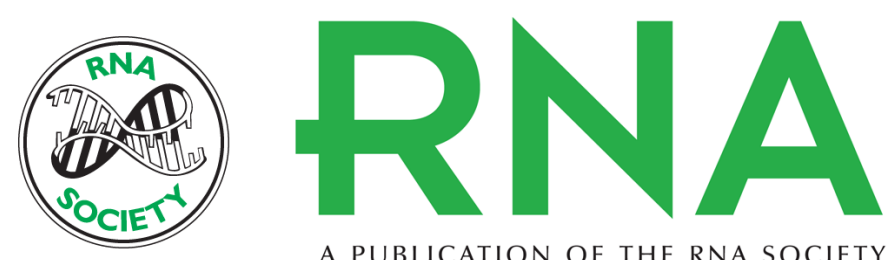

A PUBLICATION OF THE RNA SOCIETY

\section{G-ribo motif favors the formation of pseudoknots in ribosomal RNA}

Sergey V. Steinberg and Yury I. Boutorine

RNA 2007 13: 1036-1042 originally published online May 16, 2007

Access the most recent version at doi:10.1261/rna.495207

$\begin{array}{ll}\text { References } & \begin{array}{l}\text { This article cites } 21 \text { articles, } 6 \text { of which can be accessed free at: } \\ \text { http://rnajournal.cshlp.org/content/13/7/1036.full.html\#ref-list-1 }\end{array}\end{array}$

\section{License}

Email Alerting Receive free email alerts when new articles cite this article - sign up in the box at the Service top right corner of the article or click here.

To subscribe to $R N A$ go to:

http://rnajournal.cshlp.org/subscriptions 